\section{HETEROCYCLIC COMPOUNDS}

Advances in Heterocyclic Chemistry

Vol. 2. Edited by A. R. Katritzky, assisted by A. J. Boulton and J. M. Lagowski. Pp. xiv +459. (New York: Academic Press, Inc.; London: Academic Press, Inc. (London), Ltd., 1963.) 14 dollars.

HE promise shown by the first volume in the series Advances in Heterocyclic Chemistry has been more than maintained in the second. This is an excellent work and any adverse criticism in this review must be judged in the light of the overall high quality. It is now elear that this series will be mostly used for general reading and that its weakness as a work of reference lies in the irregular sequence of the chapters. Possibly a cumulative subject index should be considered for incorporation in a subsequent volume or volumes.

As in Volume 1, approaches have been made in several ways. Thus, some chapters are based on the type of heterocyclic nucleus; some deal with a particular reagent and its effects on heterocyclic compounds in general; and others are highly specialized, dealing with a particular reaction of a particular class of heterocycle. In addition the editor and one of the assistant editors have contributed two more chapters on prototropic tautomerism. Of the chapters of the first type, one on three-membered rings with two hetero-atoms comprises an excellent account of the chemistry of the oxiranes, the diaziridines and the diazirines, to the last two of which the author, E. Schmitz, has made a considerable personal contribution. These fascinating small-ring systems were unknown before 1950 , although in earlier days nitrones, hydrazones and aliphatic diazo compounds had all been erroneously formulated as such. The contrast between the properties of the diazirines and their linear isomers, the diazoalkanes is partioularly interesting. Thus the eyclic compounds are much more stable (except possibly in the condensed state) and much less reactive.

Quinoxalines and isoxazoles, much better known heterocyclic classes, are the subjects of chapters by G. W. H. Cheeseman and by N. K. Kochetkov and S. D. Sokolov respectively. The translation from the Russian of the latter by A. L. Pumpiansky is worthy of the highest praise. Both are first-rate reviews of the work that has been done on these compounds in the past eight to ten years. A great deal of interest has centred on the $N$-oxides of the quinoxalines because of the rearrangements and reactions they undergo and their biological activity. For the rest, the quinoxalines fall neatly into place in the general scheme of aza-naphthalene chemistry. The isoxazoles, on the other hand, are characterized by a propensity to undergo ring fission on nucleophilie attack.

An even less-stable nucleus is that of the selenazoles. Difficulties in synthesis and toxicity of some members have, in addition, led to this group of compounds being little investigated. E. Bulka has provided an interesting account of this little-known subject. The chapter on 1,3-oxazines will be found of less general interest, since the only compounds of authentic structure are all hydrohetero-aromatic and consequently their properties are largely those that could be forecast from an investigation of their open-chain analogues-thus, typically, tetrahydro-1,3-oxazines are cyclic aldehyde-ammonias or carbamates and dihydro-1,3-oxazines are cyclic isoureas or isoamides.

There are two chapters on reactions of hetero-aromatic compounds with particular reagents. That by $R$. O. C. Norman and G. K. Radda, who have given a general account of the reactions with free radicals, is most informative. The surprising power of orientation of the heteroatom or atoms is well emphasized in the text and in a table which gives examples of the products of reaction of aryl radicals with, among others, pyridine, quinoline, pyridazine and quinoxaline. Reactions with diazo- methane are the subject of the chapter by R. Gompper, who has covered a most diffuse-topic in masterly fashion. His approach throughout has been to tie together his account of the multitudinous reactions of diazomethane with heterocyclic compounds with a strong throad of theory of reaction mechanisms. In the longest section, which is devoted to the methylation of cyclic lactams, the large measure of correlation between the point of attack $(\mathrm{O}$ or $\mathrm{N})$ and the amide-carbonyl stretching frequency is clearly demonstrated.

Those readers who are interested in the highly specialized subjects of the action of metal catalysts on pyridine and the polymerization of pyrroles and indoles are well catered for in excellent reviews by G. M. Badger and W. H. F. Sasse and by G. F. Smith respectively.

A. R. Katritzky and J. M. Lagowski havo continued their account of prototropic tautomerism with two more chapters in this volume. Their discussion of the structures of, largely, amino and hydroxy derivatives of furan, thiophene, pyrrole, indole, pyrazole, imidazole and the like is excellent on a factual basis. The style, particularly in the second chapter, is rather tiresome and in places is little more than a list of references joined by non-committal phrases of the type: "Brown has shown that ... but Robinson has indicated that . . . whereas Jones has suggested that ...".

This is an excellent volume and can be wholeheartedly recommended for students and research workers in the $\begin{array}{ll}\text { field of organic chemistry. } & \text { A. F. Crowthen }\end{array}$

\section{MOLECULAR REARRANGEMENTS}

\section{Molecular Rearrangements}

Part 1. Edited by Paul do Mayo. Pp. xii + 706. (New York and London: Interscience Publishers, a Division of John Wiley and Sons, 1963.) 185s.

$\mathrm{T}$ HIS volume forms the first part of a comprehensive two-volume monograph of molecular rearrangements in organic chemistry. It deals mainly with the rearrangements that have been the subject of detailed mechanistic investigations and have provided the foundation of our understanding of this field; the second volume is intended to deal with some less-understood rearrangements involving steroids, terpenes, alkaloids, carbohydrates and aminoacids. The division between the volumes is not, therefore, simply based on the chemical structures involved; Part 1 includes a discussion of tho detailed mechanistic investigations involving the rearrangement reactions of bicyclic terpenes.

The scope of the work is illustrated by the following chapter headings: "Wagner-Meerwein and Pinacolic Rearrangements" (Y. Pocker); "Allylic Rearrangements" (P. D. B. de la Mare); "Carbonium Ion Pearrangements in Bridged Bicyclic Systems" (J. A.. Berson); "Rearrangements in Small Ring Compounds" (R. Breslow); "Aromatic Rearrangements" (M. J. S. Dewar); "Base-catalysed Rearrangements" (H. E. Zimmerman); "Free Radical Rearrangements" (C. Walling); "Rearrangements Involving Electron-deficient Nitrogen or Oxygen" (P. A. S. Smith); "Transannular Hydride Shifts" (V. Prelog and J. G. Traynham); "Heterocyclic Rearrangements" (G. M. Badger and. J. W. Clark-Lewis); "Claisen and Related Rearrangements" (Sara J. Rhoads).

The length of the chapters varios considerably, those by de la Mare, Berson, and Smith together making up almost half the book. The first of these covers a wider field than allylic rearrangements; it includes many of the data on the solvolysis of allylic compounds, together with an account of the formation of allylic carbonium ions by additions of electrophiles to dienes. The chapter provides a valuable summary of these interrelated reactions. The description of bicyclic rearrangements by Berson starts 